\title{
Oscillatory Solutions of a System of $a$ Nonlinear Integro-Differential Equations of Second Order with Deviating Arguments
}

\section{FOL TYŃSKA}

Some conditions are established under which solutions of a system of nonlinear integro-differential equations are oscillatory.

Koy words: Nonlinear integro-differential equations, equations of second order, oscillatory solutions

AMS subject classification: $45 \mathrm{~J} 05,45 \mathrm{M} 99$

\section{Introduction}

Consider the system of equations

$$
y_{j}^{\prime \prime}(t)=\int_{0}^{t} f_{i}\left(t, s ; y_{1}(s), \ldots, y_{n}(s) ; y_{1}\left(g_{1}(s)\right), \ldots, y_{n}\left(g_{n}(s)\right)\right) d s \quad(i=1, \ldots, n)
$$

where

$$
f_{j}: \mathbb{R}_{+}^{2} \times \mathbf{R}^{n} \times \mathbf{R}^{n} \rightarrow \mathbf{R} \quad \text { and } \quad g_{k}: \mathbf{R}_{+} \rightarrow \mathbb{R}_{+}(k=1, \ldots, n)
$$

are continuous functions and

$$
g_{k}(t) \leq t \text { or } g_{k}(t)>t \text { for all } t \text { and } \lim _{t \rightarrow \infty} g_{k}(t)=\infty \quad(k=1, \ldots, n) .
$$

Sufficient conditions under which every solution of the system (1) is oscillatory will be established. Both cases of retarded and advanced arguments will be considered. A similar problem for the system of $n$ nonlinear integro-differential equations of the first order have been considered in [1] and [2]. We shall use a method of proofs similar to that given in [4] for the system of $n$ ordinary differential equations with delayed arguments.

\section{Preliminaries}

By a solution $y=\left\langle y_{1}, \ldots, y_{n}\right\rangle$ of the system (1) we shall understand only a non-trivial solution extended to the infinity. A solution $y$ of the system (1) is called

a) oscillatory, if every component $y_{k}$ of $y$ has an infinite sequence of zeros tending to infinity as the argument tends to infinity.

b) non-oscillatory, if every component $y_{k}$ of $y$ has a constant sign for sufficiently large values of the argument $t$, i.e. for $t \geq T$, for some $T \geq 0$. 
We shall use the following Assumptions:

(i) $g_{k}(k=1, \ldots, n)$ are continuous and non-decreasing functions.

(ii) $f_{i}\left(t, s ; y_{1}, \ldots, y_{n} ; u_{1}, \ldots, u_{n}\right) \operatorname{sign} u_{i+1} \geq a_{j}(t, s)\left|H_{i}\left(y_{i+1}\right)\right|(i=1, \ldots, n-1)$ $f_{n}\left(t, s ; y_{1}, \ldots, y_{n} ; u_{1}, \ldots, u_{n}\right) \operatorname{sign} u_{1} \leq-a_{n}(t, s)\left|H_{n}\left(u_{1}\right)\right|$ for all $\left(t, s ; y_{1}, \ldots, y_{n} ; u_{1}, \ldots, u_{n}\right) \in \mathbf{R}_{+} \times \mathbf{R} \times \mathbf{R}$, where $H_{i}: \mathbf{R} \rightarrow \mathbb{R}$ are continuous and non-decreasing functions, $H_{i}\left(u_{i+1}\right) u_{i+1}>0, u_{i+1} \in \mathbb{R}(i=1, \ldots, n), u_{n+1}=u_{i}$ and $a_{i}: \mathbb{R}_{+}$ $\times \mathbf{R}_{+} \rightarrow \mathbf{R}$ are continuous functions, $a_{i}(t, s)>0$ for all $(t, s) \in \mathbf{R}_{+} \times \mathbb{R}_{+}(i=1, \ldots, n)$.

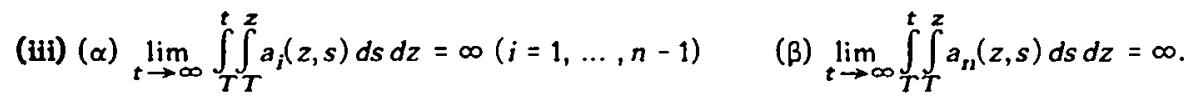

(iv) $F_{i}:=\int_{0}^{T} f_{i}\left(\cdot, s ; y_{1}(s), \ldots, y_{n}(s) ; y_{1}\left(g_{1}(s)\right), \ldots, y_{n}\left(g_{n}(s)\right)\right) d s \in L_{1}[a, \infty)(i=1, \ldots, n)$

for a constant $a>0$, where $\left|F_{i}(t)\right| \leq K_{i}$ for some constants $K_{i}$ and $F_{i}(t) y_{i+1}\left(t+t_{0}\right)>0$ $(i=1, \ldots, n-1), F_{n}(t) y_{1}\left(t+t_{0}\right)<0$ for some $t_{0}>0, t \in \mathbb{R}_{+}\left(y_{n+1}=y_{1}\right)$.

In addition, we shall use the notations

$$
M_{i}=\int_{T}^{\infty}\left|F_{i}(t)\right| d t \quad \text { and } N_{i}=\inf _{\left[T_{i}, \infty\right)}\left|H_{i}\left(u_{i}\right)\right| \text { for some } T_{i}>0 \quad(i=1, \ldots, n) .
$$

\section{Main results (the case $g_{k}(t) \leq t$ )}

First we shall consider the system (1) with retarded arguments.

Lemma 1: Let the assumptions (i), (ii) and (iv) hold and let $y$ be a solution of the system (1). If one of its components is non-oscillatory, then $y$ itself is non-oscillatory and monotonic.

Proof: Let $y_{i}$ be a non-oscillatory component of $y$. For the proof let be $y_{j}(t)>0$ for $t \geq t_{0} \geq$ 0 and $y_{i}\left(g_{i}(t)\right)>0$ for $t \geq T_{0}$, where $T_{0} \geq t_{0}$. From the system (1) and the assumptions (ii), (iv) it follows $\left(y_{0}=y_{n+1}, t_{0}=t_{n}\right)$

$$
\begin{aligned}
y_{i-1}^{\prime \prime}(t) & =\int_{0}^{t} f_{i-1}\left(t, s ; y_{1}(s), \ldots, y_{n}(s) ; y_{1}\left(g_{1}(s)\right), \ldots, y_{n}\left(g_{n}(s)\right)\right) d s \\
& =\int_{0}^{T_{0}} f_{i-1}\left(t, s ; y_{1}(s), \ldots, y_{n}(s) ; y_{1}\left(g_{1}(s)\right), \ldots, y_{n}\left(g_{n}(s)\right)\right) d s \\
& =+\int_{T_{0}}^{t} f_{i-1}\left(t, s ; y_{1}(s), \ldots, y_{n}(s) ; y_{1}\left(g_{1}(s)\right), \ldots, y_{n}\left(g_{n}(s)\right)\right) \operatorname{sign} y_{i}\left(g_{i}(s)\right) d s \\
& 2 F_{i-1}(t)+\int_{T_{0}}^{t} a_{i-1}(t, s)\left|H_{j-1}\left(y_{j}(s)\right)\right| d s>0 .
\end{aligned}
$$

Hence $y_{j-1}^{\prime}$ and $y_{j-1}$ are monotonic functions and, for sufficiently large $t$ (for example for $t$ $\left.\geq t_{1}, t_{1} \geq t_{0}\right)$, they have the constant sign. Now $y_{i-1}$ may be a positive or negative function. Let be $y_{i-1}(t)<0$ for $t \geq t_{1}$ and $y_{i-1}\left(g_{j-1}(t)\right)<0$ for $t \geq T_{1} \geq t_{1}$. Hence from the system (1) and the assumptions (ii), (iv) we get 


$$
\begin{aligned}
y_{i-2}^{\prime \prime}(t)= & \int_{0}^{T_{1}} f_{i-2}\left(t, s ; y_{1}(s), \ldots, y_{n}(s) ; y_{1}\left(g_{1}(s)\right), \ldots, y_{n}\left(g_{n}(s)\right)\right) d s \\
& -\int_{T_{1}}^{t} f_{i-2}\left(t, s ; y_{1}(s), \ldots, y_{n}(s) ; y_{1}\left(g_{1}(s)\right), \ldots, y_{n}\left(g_{n}(s)\right)\right) \operatorname{sign} y_{j-1}\left(g_{i-1}(s)\right) d s \\
& \leq F_{i-2}(t)-\int_{\frac{T}{1}}^{t} a_{i-2}(t, s)\left|H_{i-2}\left(y_{i-1}(s)\right)\right| d s<0
\end{aligned}
$$

or if $y_{i-1}(t)>0$ for $t \geq t_{1}$ and $y_{i-1}\left(g_{i-1}(t)\right)>0$ for $t \geq T_{1}$, then, from the system (1) and the assumptions (ii), (iv),

$$
\begin{aligned}
y_{i-2}^{\prime \prime}(t) & =\int_{0}^{T_{1}} f_{i-2}\left(t, s ; y_{1}(s), \ldots, y_{n}(s) ; y_{1}\left(g_{1}(s)\right), \ldots, y_{n}\left(g_{n}(s)\right)\right) d s \\
& =+\int_{T_{1}}^{t} f_{i-2}\left(t, s ; y_{1}(s), \ldots, y_{n}(s) ; y_{1}\left(g_{1}(s)\right), \ldots, y_{n}\left(g_{n}(s)\right)\right) \operatorname{sign} y_{i-1}\left(g_{i-1}(s)\right) d s \\
& \geq F_{i-2}(t)+\int_{T_{1}}^{t} a_{i-2}(t, s)\left|H_{i-2}\left(y_{i-1}(s)\right)\right| d s>0 .
\end{aligned}
$$

In both cases it follows that $y_{i-2}^{\prime}$ and $y_{i-2}$ are monotonic functions and for sufficiently large $t$ they have constant sign (for example for $t z t_{2}$ ). Proceeding in the same way we obtain $y_{j}, y_{j-1}$, $y_{1}, \ldots, y_{i+1}$ for $t \geq T_{i} \geq t_{i}(i=1, \ldots, n)$ are monotonic and therefore non-oscillatory functions

Corollary 1: Under the assumptions of Lemma 1, if one of the components of the solutuion $y$ of the system (1) is oscillatory, then the solution itself is oscillatory.

Theorem 1: If the conditions (i) - (iv) hold, then every bounded solution of the system (1) is oscillatory.

Proof: Suppose that there exists a bounded and non-oscillatory solution $y=\left\langle y_{1}, \ldots, y_{n}\right\rangle$ of the system (1). Let $y_{j+1}(t)>0$ for $t \geq t_{0} \geq 0$ and $\left|y_{i}(t)\right| \leq Q$ for $t \geq t_{0}(i=1, \ldots, n), Q$ being same constant and $y_{n+1}=y_{1}$. Then $y_{i+1}\left(g_{i+1}(t)\right)>0$ for $t \geq T_{0}, T_{0} \geq t_{0}$ being some constant. By system (1) and the assumptions (ii), (iv) we have

$$
\begin{aligned}
y_{i}^{\prime \prime}(t)= & \int_{0}^{T_{0}} f_{i}\left(t, s ; y_{1}(s), \ldots, y_{n}(s) ; y_{1}\left(g_{1}(s)\right), \ldots, y_{n}\left(g_{n}(s)\right)\right) d s \\
& +\int_{t_{0}}^{t} f_{i}\left(t, s ; y_{1}(s), \ldots, y_{n}(s) ; y_{1}\left(g_{1}(s)\right), \ldots, y_{n}\left(g_{n}(s)\right)\right) \operatorname{sign} y_{i+1}\left(g_{i+1}(s)\right) d s \\
z & F_{j}(t)+\int_{T_{0}^{t}}^{t} a_{i}(t, s)\left|H_{j}\left(y_{i+1}(s)\right)\right| d s>0 .
\end{aligned}
$$

Hence

$$
y_{i}^{\prime \prime}(t) \geq F_{i}(t)+\int_{T_{0}}^{t} a_{j}(t, s)\left|H_{i}\left(y_{i+1}(s)\right)\right| d s .
$$


Integrating (2) from $T_{0}$ to $t$ and using (iv) we obtain

$$
y_{j}^{\prime}(t) \geq y_{j}^{\prime}\left(T_{0}\right)+\int_{T_{0}}^{t}\left|F_{j}(s)\right| d s+N_{i} \int_{\tau_{0}}^{t} \int_{T_{0}}^{z} a_{j}(z, s) d s d z>0 .
$$

If $y_{i}^{\prime \prime}(t)>0$ and $y_{i}^{\prime}(t)>0$, then $y_{i}(t) \rightarrow \infty$ as $t \rightarrow \infty$. This is a contradiction with the supposition that the solution of the system (1) is bounded. Therefore every bounded solution of the system (1) is oscillatory

Lemma 2: If the assumptions (i) $-(\mathrm{iii}) /(\alpha)$ and (iv) are satisfied, then all components $y_{i}$ $(i=1, \ldots, n)$ of a non-oscillatory solution $y$ of the system (1) have the same sign for sufficiently large $t$.

Proof: We shall consider the two cases a) $i=n$ and b) $i \neq n$.

a) The case $i=n$. Then $y_{n}$ is a non-oscillatory component. Let for the proof $y_{n}(t)>0$ for $t$ $2 t_{n-1}$. Then $y_{n}\left(g_{n}(t)\right)>0$ for $t>T_{n-1} \geq t_{n-1}\left(t_{n-1}, T_{n-1}=\right.$ const 20$)$. We shall show that the remaining components are positive. From system (1) and the assumptions (ii), (iv) we have

$$
y_{n-1}^{\prime \prime}(t) \geq F_{n-1}(t)+\int_{T_{n-1}}^{t} a_{n-1}(t, s) \mid H_{n-1}\left(y_{n}((s)) \mid d s>0 .\right.
$$

Hence $y_{n-1}^{\prime}$ is an increasing function and for sufficiently large $t$ it has constant sign. Integrating (3) from $T_{n-1}$ to $t$ we obtain

$$
\begin{aligned}
y_{n-1}^{\prime}(t) & \geq y_{n-1}^{\prime}\left(T_{n-1}\right)+\int_{T_{n-1}}^{t}\left|F_{n-1}(s)\right| d s+\int_{T_{n-1}}^{t} \int_{T_{n-1}}^{z} a_{n-1}(z, s) \mid H_{n-1}(y((s)) \mid d z d s \\
& \geq c_{0}+M_{n-1}+N_{n-1} \int_{T_{n-1}}^{t} \int_{T_{n-1}}^{z} a_{n-1}(z, s) d z d s, \quad c_{0}=y_{n-1}^{\prime}\left(T_{n-1}\right)=\text { const. }
\end{aligned}
$$

Hence by assumption (iii) $/(\alpha) y_{n-1}^{\prime}(t)>0$. If $y_{n-1}^{\prime \prime}(t)>0$ and $y_{n-1}^{\prime}(t)>0$, then $y_{n-1}(t) \rightarrow \infty$ as $t$ $\rightarrow \infty$. In this way we can prove that, for $t \geq T_{n} \geq t_{n} \geq 0, y_{i}(t)>0(i=1, \ldots, n)$. (If we suppose that $y_{n}(t)<0$, then $y_{j}(t)<0$ for $i=1, \ldots, n, t \geq T_{n} \geq t_{n} \geq 0$.)

b) The case $i \neq n$. Then $y_{i}(t)>0$ for $t \geq t_{j} \geq 0$ and $y_{j}\left(g_{j}(t)\right)>0$ for $t \geq T_{j} \geq t_{i}$. We shall show that $y_{i+1}(t)>0$ for $t \geq t_{i}$. Suppose conversely that $y_{i+1}(t)<0$ for $t \geq t_{i+1}$ and $y_{i+1}\left(g_{i+1}(t)\right)<0$ for $t \geq T_{j+1} \geq t_{j+1}$. From the $i$-th equation of the system (1) and the assumptions (ii), (iv) we have

$$
y_{i}^{\prime \prime}(t) \leq F_{i}(t)-\int_{T_{i+1}}^{t} a_{i}(t, s)\left|H_{j}\left(y_{i+1}(s)\right)\right| d s \leq F_{i}(t)-N_{i} \int_{T_{i+1}}^{t} a_{i}(t, s) d s<0 .
$$

Integrating (4) from $T_{i+1}$ to $t$ we have

$$
y_{i}^{\prime}(t) \leq y_{i}^{\prime}\left(T_{i+1}\right)-\int_{i+1}^{t}\left|F_{i}(s)\right| d s-N_{i} \int_{T_{i+1}}^{t} \int_{T_{i+1}}^{z} a_{i}(z, s) d s d z=C_{0}-M_{i}-N_{i} \int_{T_{i+1}}^{t} \int_{T_{i+1}+a_{i}}^{z} a_{i}(z, s) d s d z
$$

Hence by (iii) $/(\alpha) y_{i}^{\prime}(t)<0$. If $y_{i}^{\prime \prime}(t)<0$ and $y_{i}^{\prime}(t)<0$, then $y_{i}(t)<0$ for sufficiently large $t$. 
This is a contradiction to the supposition $y_{j}(t)>0$. Proceeding in the same way we may show that $y_{i}(t)>0$ for $i=1, \ldots, n-1$ and for sufficiently large $t$.

Suppose that $y_{n}(t)<0$ for $t \geq t_{n-1}$ and then $y_{n}\left(g_{n}(t)\right)<0$ for $t \geq T_{n-1} \geq t_{n-1}$. From the ( $n$ -1) -th equation of the system (1) and the assumptions (ii), (iv) we get

$$
y_{n-1}^{\prime \prime}(t) \leq F_{n-1}(t)-\int_{T_{n-1}}^{t} a_{n-1}(t, s)\left|H_{n-1}\left(y_{n}(s)\right)\right| d s<0 .
$$

Integrating (5) from $T_{n-1}$ to $t$ we get

$$
y_{n-1}^{\prime}(t) \leq y_{n-1}^{\prime}\left(T_{n-1}\right)-\int_{T_{n-1}^{\prime}}^{t}\left|F_{n-1}(s)\right| d s-N_{n-1} \int_{T_{n-1}}^{t} \int_{T_{n-1}}^{z} a_{n-1}(z, s) d s d z .
$$

_Hence by assumption (iii) $/(\alpha) y_{n-1}^{\prime}(t)<0$. If $y_{n-1}^{\prime \prime}(t)<0$ and $y_{n-1}^{\prime}(t)<0$, then $y_{n-1}(t)<0$ for sufficiently large $t$. This is a contradiction to $y_{n-2}(t)>0$, which was shown early. Therefore $y_{n}(t)>0$ and $y_{j}(t)>0$ for $i=1, \ldots, n$

Theorem 2: Let the assumptions (i) - (iv) be satisfied and

$$
\lim _{t \rightarrow \infty} \int_{T}^{t} a_{n}(z, s) d s=\infty .
$$

Then all solutions of the system (1) are oscillatory.

Proof: Suppose that $y_{1}$ is a non-oscillatory component of the solution $y$ of (1) and let $y_{1}(t)$ $>0$ for $t \geq t_{0}$. Then $y_{1}\left(g_{1}(t)\right)>0$ for $t \geq T_{0} \geq t_{0}$. By Lemma 1 all components $y_{j}(i=1, \ldots, n)$ are non-oscillatory and monotonic functions. Moreover by Lemma 2 it follows that all non-oscillatory components have the same sign for sufficiently large $t$. Suppose that all components are positive. (In the case that all components are negative - the proof is analogous.) Then by (6) from the last equation of the system (1) and the assumptions (ii), (iv) it follows that

$$
y_{n}^{\prime \prime}(t) \leq F_{n}(t)-\int_{T_{n}}^{t} a_{n}(t, s)\left|H_{n}\left(y_{1}\left(g_{1}(s)\right)\right)\right| d s \leq K_{n}-N_{n} \int_{T_{n}}^{t} a_{n}(t, s) d s,
$$

where $K_{n}$ is a constant (see (iv)). By assumption (6) $y_{n}^{\prime \prime}(t)<0$. Moreover

$$
y_{n}^{\prime \prime}(t) \leq F_{n}(t)-N_{n} \int_{n}^{t} a_{n}(t, s) d s .
$$

Integrating (7) from $T_{n}$ to $t$ and by assumption (iv) we have

$$
y_{n}^{\prime}(t) \leq y_{n}^{\prime}\left(T_{n}\right)+M_{n}-N_{n} \int_{T_{n}}^{t} \int_{T_{n}}^{z} a_{n}(z, s) d s d z .
$$

By assumption (iii) $/(\beta) y_{n}^{\prime}(t)<0$. If $y_{n}^{\prime \prime}(t)<0$ and $y_{n}^{\prime}(t)<0$, then $y_{n}(t)<0$ for sufficiently large $t$. This contradiction proves that the component $y_{1}$ is oscillatory. By Corollary 1 , if $y_{1}$ is an oscillatory component, then all components of the solution $y$ of the system (1) are oscillatory functions 


\section{Main results (the case $g_{k}(t)>t$ )}

In the case of advanced arguments our lemmas and theorems have the following form.

Lemma 1': Let the assumptions (i) and (ii) be satisfied and let $y$ be a solution of the system (1). If one of its components is non-oscillatory, then $y$ itself is non-oscillatory and monotonic.

Lemma $2^{\prime}:$ If the assumptions $(\mathrm{i})-(\mathrm{iii}) /(\alpha)$ are satisfied, then all components $y_{i}(i=1, \ldots$. $, n)$ of a non-oscillatory solution $y$ of the system (1) have the same sign for sufficiently large $t$.

Theorem 1': If the assumptions (i) - (iii) are satisfied, then every bounded solution of the system (1) is oscillatory.

Theorem 2': Let the assumptions (i) - (iii) and (6) be satisfied. Then all solutions of the system (1) are oscillatory.

The proofs of these statements are similar as in the case $g_{k}(t) \leq t$.

\section{REFERENCES}

[1] FOLTYŃSKA, I.: Oscillatory solutions of the system of $n$ integro-differential equations with deviating arguments. Funct. et Appr. 19 (1990), 97 - 105.

[2] FOLTYŃSKA, I.: Oscillatory property for the system of $n$ nonlinear integro-differential equations with deviating arguments. Submitted.

[3] FOLTYŃSKA, I.: Oscillation of solution to a system. of nonlinear integro-differential equations. Demonstr. Math. 18 (1985), 763 - 775.

[4] VARECH, N.V. and N.V. SHEVELO: On the conditions of the oscillation of the solutions to systems of differential equations with retarded arguments. In: The Qualitative Methods of the Theory of Differential Equations with Deviating Arguments. Inst. Math. Acad. Sci. USSR (Kiev) 1977, pp. 26 - 44 (in Russian).

Dr. Izabella Foltyriska

Technical University of Poznan

Institute of Mathematics

ul. Piotrowo 3 a

60-965 Poznań

Poland 